CRYSTALLOGRAPHIC COMMUNICATIONS

ISSN 2056-9890

Received 6 November 2015

Accepted 24 November 2015

Edited by $\mathrm{H}$. Stoeckli-Evans, University of Neuchâtel, Switzerland

Keywords: crystal structure; 8-quinolinol; bis(2-picolyl)amine; hydrogen bonding; $\pi-\pi$ interactions

CCDC reference: 1438483

Supporting information: this article has supporting information at journals.iucr.org/e

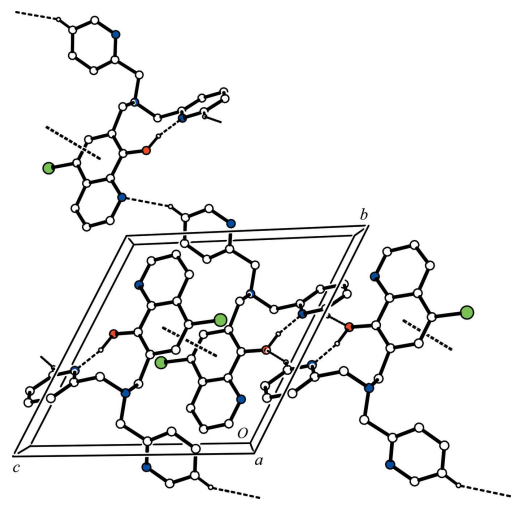

OPEN $\odot$ ACCESS

\section{Crystal structure of 7-\{[bis(pyridin-2-ylmethyl)- amino]methyl\}-5-chloroquinolin-8-ol}

\author{
Koji Kubono, ${ }^{\text {a* Kimiko Kado, }}{ }^{\text {a }}$ Yukiyasu Kashiwagi, ${ }^{\mathrm{b}}$ Keita Tani $^{\mathrm{a}}$ and Kunihiko \\ Yokoi ${ }^{\mathrm{a}}$ \\ ${ }^{\mathbf{a} D}$ Division of Natural Sciences, Osaka Kyoiku University, Kashiwara, Osaka 582-8582, Japan, and ${ }^{\mathbf{b}}$ Osaka Municipal \\ Technical Research Institute, Osaka 536-8553, Japan. *Correspondence e-mail: kubono@cc.osaka-kyoiku.ac.jp
}

In the title compound, $\mathrm{C}_{22} \mathrm{H}_{19} \mathrm{ClN}_{4} \mathrm{O}$, the quinolinol moiety is almost planar [r.m.s. deviation $=0.012 \AA$ ] . There is an intramolecular $\mathrm{O}-\mathrm{H} \cdots \mathrm{N}$ hydrogen bond involving the hydroxy group and a pyridine $\mathrm{N}$ atom forming an $S(9)$ ring motif. The dihedral angles between the planes of the quinolinol moiety and the pyridine rings are 44.15 (9) and $36.85(9)^{\circ}$. In the crystal, molecules are linked via $\mathrm{C}-\mathrm{H} \cdots \mathrm{O}$ hydrogen bonds forming inversion dimers with an $R_{4}^{4}(10)$ ring motif. The dimers are linked by $\mathrm{C}-\mathrm{H} \cdots \mathrm{N}$ hydrogen bonds, forming ribbons along [011] ]. The ribbons are linked by $\mathrm{C}-\mathrm{H} \cdots \pi$ and $\pi-\pi$ interactions [intercentroid distance $=3.7109(11) \AA$ ] , forming layers parallel to $(01 \overline{1})$.

\section{Chemical context}

8-Quinolinol and its derivatives are well-known chelating reagents, forming fluorescent complexes with various metal ions, such as $\mathrm{Al}^{3+}, \mathrm{Zn}^{2+}$ and $\mathrm{Cd}^{2+}$ (Goon et al., 1953; Valeur \& Leray, 2000; Pohl \& Anzenbacher, 2003). Bis(pyridin-2ylmethyl)amine [di-(2-picolyl)amine (DPA)] is an excellent ligand showing high selectivity for $\mathrm{Zn}^{2+}$, which plays important roles in biological, pathological and environmental processes (Berg \& Shi, 1996; Bush et al., 1994; Callender \& Rice, 2000), and it is used to detect $\mathrm{Zn}^{2+}$ with low concentration in biological and environmental samples. Therefore, many fluorescence probes for $\mathrm{Zn}^{2+}$ bearing DPA as an ion-recognition site have been developed (Xue et al., 2008; Chen et al., 2011; Kwon et al., 2012). We have synthesized a new fluorescence chemosensor, based on 8-quinolinol containing DPA via a two-step reaction, and herein we report on its synthesis and crystal structure.

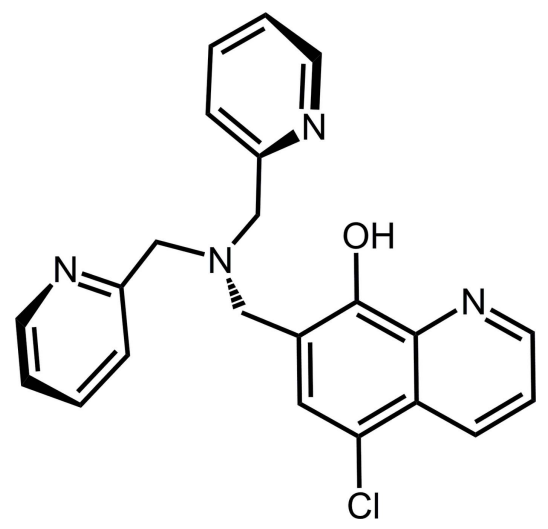

\section{Structural commentary}

The molecular structure of the title compound, is shown in Fig. 1. There is an $\mathrm{O}-\mathrm{H} \cdots \mathrm{N}$ intramolecular hydrogen bond 


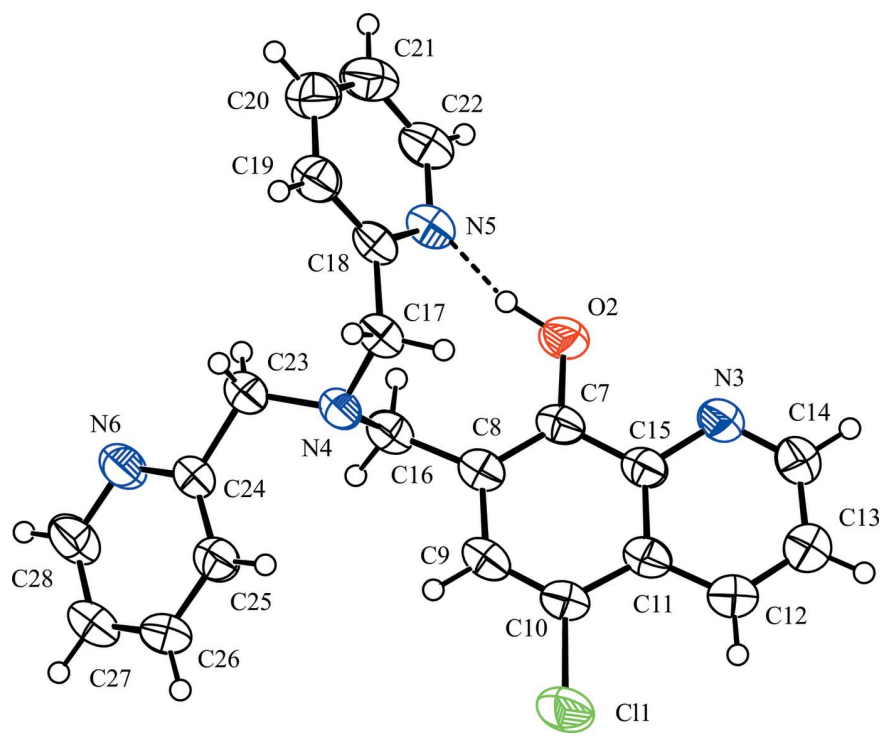

Figure 1

The molecular structure of the title compound, showing the atom labelling. Displacement ellipsoids are drawn at the $50 \%$ probability level. The intramolecular $\mathrm{O}-\mathrm{H} \cdots \mathrm{N}$ hydrogen bond is shown as a dashed line (see Table 1).

involving the hydroxy group $(\mathrm{O} 2-\mathrm{H} 2)$ and a pyridine $\mathrm{N}$ atom, $\mathrm{N} 5$, generating an $S(9)$ ring motif (Fig. 1 and Table 1). The $\mathrm{N}$ (tertiaryamine) $-\mathrm{C}-\mathrm{C}-\mathrm{N}$ (pyridine) torsion angles, $\mathrm{N} 4-$ $\mathrm{C} 17-\mathrm{C} 18-\mathrm{N} 5$ and $\mathrm{N} 4-\mathrm{C} 23-\mathrm{C} 24-\mathrm{N} 6$ are 75.0 (2) and $152.46(19)^{\circ}$, respectively. The dihedral angle between the N5and N6-containing pyridine rings pyridine rings is $80.97(12)^{\circ}$,

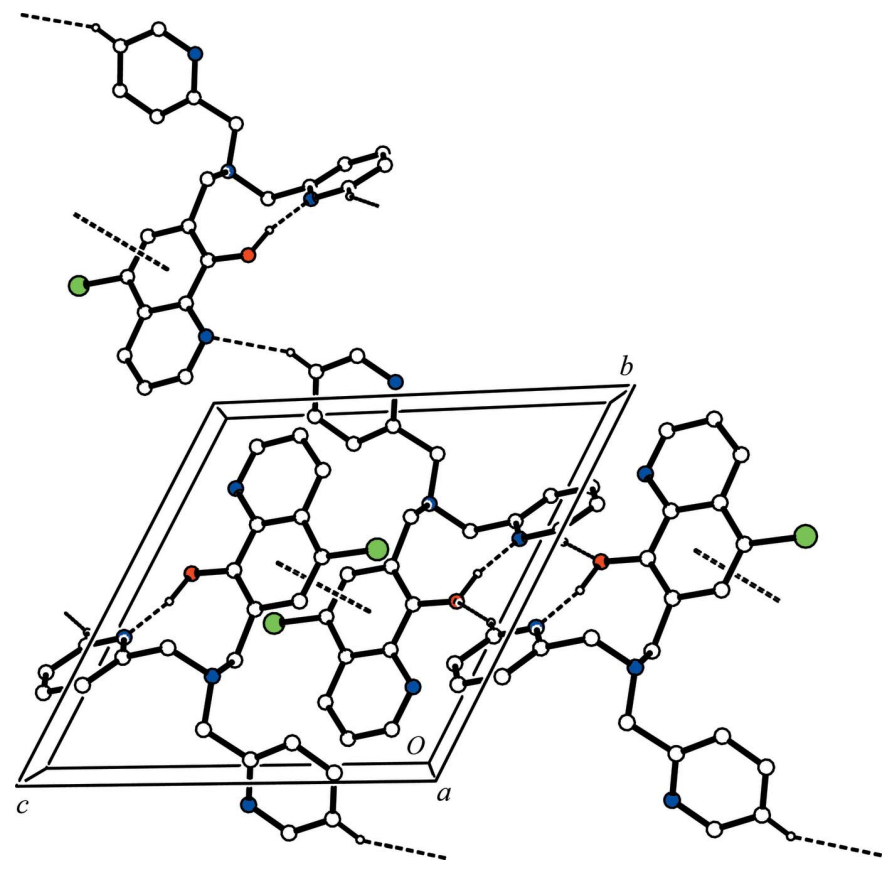

Figure 2

A view along the $a$ axis of the crystal packing of the title compound. The hydrogen bonds (see Table 1 ) and $\pi-\pi$ interactions are shown as dashed lines. $\mathrm{H}$ atoms not involved in these interactions have been omitted for clarity.
Table 1

Hydrogen-bond geometry $\left(\AA,^{\circ}\right)$.

$\mathrm{Cg} 2$ and $\mathrm{Cg} 3$ are the centroids of rings N5/C18-C22 and N6/C24-C28, respectively.

\begin{tabular}{lllll}
\hline$D-\mathrm{H} \cdots A$ & $D-\mathrm{H}$ & $\mathrm{H} \cdots A$ & $D \cdots A$ & $D-\mathrm{H} \cdots A$ \\
\hline $\mathrm{O} 2-\mathrm{H} 2 \cdots \mathrm{N} 5$ & $1.04(3)$ & $1.66(4)$ & $2.689(3)$ & $168(2)$ \\
$\mathrm{C} 22-\mathrm{H} 22 \cdots \mathrm{O} 2^{\mathrm{i}}$ & 0.93 & 2.46 & $3.348(3)$ & 160 \\
$\mathrm{C} 27-\mathrm{H} 27 \cdots \mathrm{N} 3^{\mathrm{ii}}$ & 0.93 & 2.55 & $3.406(3)$ & 153 \\
$\mathrm{C} 17-\mathrm{H} 17 \mathrm{~b} \cdots C g 2^{\mathrm{iii}}$ & 0.97 & 2.79 & $3.599(3)$ & 141 \\
$\mathrm{C} 23-\mathrm{H} 23 A \cdots C g 3^{\text {iv }}$ & 0.97 & 2.86 & $3.770(3)$ & 156 \\
\hline
\end{tabular}

Symmetry codes: (i) $-x+1,-y+1,-z$; (ii) $x, y+1, z+1$; (iii) $-x,-y+1,-z$; (iv) $-x,-y+2,-z+1$.

and they make dihedral angles of $44.15(9)$ and $36.85(9)^{\circ}$, respectively, with the quinolinol moiety.

\section{Supramolecular features}

In the crystal, molecules are linked via $\mathrm{C}-\mathrm{H} \cdots \mathrm{O}$ hydrogen bonds, forming inversion dimers with an $R_{4}^{4}(10)$ ring motif (Fig. 2 and Table 1). The dimers are linked by $\mathrm{C}-\mathrm{H} \cdots \mathrm{N}$ hydrogen bonds, forming ribbons along [011] . The ribbons are linked by $\mathrm{C}-\mathrm{H} \cdots \pi$ (Table 1 ) and slipped parallel $\pi-\pi$ interactions $\left[C g 1 \cdots C g 1^{\mathrm{i}},=3.7109(11) \AA\right.$; $C g 1$ is the centroid of ring C7-C11/C15; inter-planar distance $=3.5518$ (8) $\AA$; slippage $=1.075 \AA$; symmetry code: (i) $-x,-y+1,-z]$, forming layers parallel to $(01 \overline{1})$.

\section{Database survey}

A search of the Cambridge Structural Database (CSD, Version 5.36; Groom \& Allen, 2014) for 8-quinolinols gave 387 hits, and for DPA, bis(pyridine-2-ylmethyl)amine gave 4535 hits. A search for the fragment 2-[bis(pyridin-2-ylmethylamino)-methyl]phenol gave 56 hits of which none contained 8-quinolinol. In the compounds that resemble the title compound, namely 2,6-bis[bis(pyridine-2-ylmethyl)aminomethyl]-4-tert-butylphenol (I) (Bjernemose \& McKenzie, 2003), and 3-\{[bis(pyridin-2-ylmethyl)amino]methyl\}-2-hydroxy-5-methylbenzaldehyde (II) (Wang et al., 2012), an intramolecular bifurcated hydrogen bond is formed. The $\mathrm{N}-$ $\mathrm{C}-\mathrm{C}-\mathrm{N}$ torsion angles in the related compounds are $-46.9(2)$ and $152.7(2)^{\circ}$ in (I) and $48.35(18)$ and $-116.99(15)^{\circ}$ in (II), compared to $75.0(2)$ and $152.46(19)^{\circ}$ in the title compound. The crystal structures of other compounds containing a fluorescent core and bis(pyridine-2-ylmethyl)amine have been reported; for example one containing a fluorescein core (Wong et al., 2009), and another a coumarin core (Kobayashi et al., 2014).

\section{Synthesis and crystallization}

A suspension of paraformaldehyde $(0.41 \mathrm{~g}, 14 \mathrm{mmol})$ and bis(2-pyridylmethyl)amine $(1.99 \mathrm{~g}, 10 \mathrm{mmol})$ in $100 \mathrm{ml}$ of $\mathrm{MeOH}$ was stirred for $18 \mathrm{~h}$ at room temperature. The solvent was removed under vacuum. To the product obtained was 
added $100 \mathrm{ml}$ of toluene and 5-chloro-8-quinolinol (1.80 g, $10 \mathrm{mmol}$ ), and the mixture was heated for $24 \mathrm{~h}$ at $353 \mathrm{~K}$. The solvent was removed under vacuum to give an oily product, which was crystallized from hexane-dichloromethane. The crude solid was recrystallized from acetonitrile to obtain yellow crystals of the title compound (yield 55\%; m.p. 380.4$382.6 \mathrm{~K})$. HRMS $(\mathrm{m} / \mathrm{z})$ : $[M+1]^{+}$calculated, 391.1326; found, 391.1271. Analysis calculated for $\mathrm{C}_{22} \mathrm{H}_{19} \mathrm{ClN}_{4} \mathrm{O}: \mathrm{C} 67.60, \mathrm{H}$ 4.90, N 14.33\%; found: C 67.50, H 5.01, N 14.37\%.

\section{Refinement}

Crystal data, data collection and structure refinement details are summarized in Table 2. The hydroxy $\mathrm{H}$ atom was located in a difference Fourier map and freely refined. The $\mathrm{C}$-bound $\mathrm{H}$ atoms were positioned geometrically and refined using a riding model: $\mathrm{C}-\mathrm{H}=0.93-0.97 \AA$ with $U_{\text {iso }}(\mathrm{H})=1.2 U_{\text {eq }}(\mathrm{C})$.

\section{Acknowledgements}

This study was supported financially in part by Grants-in-Aid for Scientific Research (No. 15 K05539) from the Japan Society for the Promotion of Science.

\section{References}

Altomare, A., Cascarano, G., Giacovazzo, C. \& Guagliardi, A. (1993). J. Appl. Cryst. 26, 343-350.

Berg, J. M. \& Shi, Y. (1996). Science, 271, 1081-1085.

Bjernemose, J. K. \& McKenzie, C. J. (2003). Acta Cryst. E59, o1275o1276.

Bush, A. I., Pettingell, W. H., Multhaup, G., d Paradis, M., Vonsattel, J.-P., Gusella, J. F., Beyreuther, K., Masters, C. L. \& Tanzi, R. E. (1994). Science, 265, 1464-1467.

Callender, E. \& Rice, K. C. (2000). Environ. Sci. Technol. 34, 232-238.

Chen, W.-H., Xing, Y. \& Pang, Y. (2011). Org. Lett. 13, 1362-1365.

Goon, E., Petley, J. E., McMullen, W. H. \& Wiberley, S. E. (1953). Anal. Chem. 25, 608-610.

Groom, C. R. \& Allen, F. H. (2014). Angew. Chem. Int. Ed. 53, 662671.

Higashi, T. (1995). ABSCOR. Rigaku Corporation, Tokyo, Japan.

Kobayashi, H., Katano, K., Hashimoto, T. \& Hayashita, T. (2014). Anal. Sci. 30, 1045-1050.

Kwon, J. E., Lee, S., You, Y., Baek, K.-H., Ohkubo, K., Cho, J., Fukuzumi, S., Shin, I., Park, S. Y. \& Nam, W. (2012). Inorg. Chem. 51, 8760-8774.
Table 2

Experimental details.

\begin{tabular}{|c|c|}
\hline \multicolumn{2}{|l|}{ Crystal data } \\
\hline Chemical formula & $\mathrm{C}_{22} \mathrm{H}_{19} \mathrm{ClN}_{4} \mathrm{O}$ \\
\hline$M_{\mathrm{r}}$ & 390.86 \\
\hline Crystal system, space group & Triclinic, $P \overline{1}$ \\
\hline Temperature (K) & 296 \\
\hline$a, b, c(\AA)$ & $8.3170(5), 11.5993(7), 11.6135$ (6) \\
\hline$\alpha, \beta, \gamma\left({ }^{\circ}\right)$ & $\begin{array}{l}116.8473(13), 105.2809(13) \\
\quad 92.0110(17)\end{array}$ \\
\hline$V\left(\AA^{3}\right)$ & $948.68(10)$ \\
\hline$Z$ & 2 \\
\hline Radiation type & Мо $K \alpha$ \\
\hline$\mu\left(\mathrm{mm}^{-1}\right)$ & 0.22 \\
\hline Crystal size $(\mathrm{mm})$ & $0.30 \times 0.20 \times 0.10$ \\
\hline \multicolumn{2}{|l|}{ Data collection } \\
\hline Diffractometer & Rigaku R-AXIS RAPID \\
\hline Absorption correction & $\begin{array}{l}\text { Multi-scan (ABSCOR; Higashi, } \\
\text { 1995) }\end{array}$ \\
\hline$T_{\min }, T_{\max }$ & $0.769,0.978$ \\
\hline $\begin{array}{l}\text { No. of measured, independent and } \\
\text { observed }\left[F^{2}>2.0 \sigma\left(F^{2}\right)\right] \text { reflec- } \\
\text { tions }\end{array}$ & $9412,4293,2329$ \\
\hline$R_{\text {int }}$ & 0.023 \\
\hline$(\sin \theta / \lambda)_{\max }\left(\AA^{-1}\right)$ & 0.648 \\
\hline \multicolumn{2}{|l|}{ Refinement } \\
\hline$R\left[F^{2}>2 \sigma\left(F^{2}\right)\right], w R\left(F^{2}\right), S$ & $0.036,0.123,1.09$ \\
\hline No. of reflections & 4293 \\
\hline No. of parameters & 257 \\
\hline $\mathrm{H}$-atom treatment & $\begin{array}{l}\mathrm{H} \text { atoms treated by a mixture of } \\
\text { independent and constrained } \\
\text { refinement }\end{array}$ \\
\hline$\Delta \rho_{\max }, \Delta \rho_{\min }\left(\mathrm{e} \AA^{-3}\right)$ & $0.26,-0.24$ \\
\hline
\end{tabular}

Computer programs: RAPID-AUTO (Rigaku, 2006), SIR92 (Altomare et al., 1993), SHELXL97 (Sheldrick, 2008), PLATON (Spek, 2009) and CrystalStructure (Rigaku, 2014).

Pohl, R. \& Anzenbacher, P. Jr (2003). Org. Lett. 5, 2769-2772.

Rigaku (2006). RAPID-AUTO. Rigaku Corporation, Tokyo, Japan. Rigaku (2014). CrystalStructure. Rigaku Corporation, Tokyo, Japan. Sheldrick, G. M. (2008). Acta Cryst. A64, 112-122.

Spek, A. L. (2009). Acta Cryst. D65, 148-155.

Valeur, B. \& Leray, I. (2000). Coord. Chem. Rev. 205, 3-40.

Wang, R.-X., Gao, D.-Z., Ye, F., Wu, Y.-F. \& Zhu, D.-R. (2012). Acta Cryst. E68, o1672-o1673.

Wong, B. A., Friedle, S. \& Lippard, S. J. (2009). J. Am. Chem. Soc. 131, 7142-7152.

Xue, L., Wang, H.-H., Wang, X. J. \& Jiang, H. (2008). Inorg. Chem. 47, 4310-4318. 


\section{supporting information}

Acta Cryst. (2015). E71, 1545-1547 [https://doi.org/10.1107/S2056989015022410]

\section{Crystal structure of 7-\{[bis(pyridin-2-ylmethyl)amino]methyl\}-5-chloro- quinolin-8-ol}

\section{Koji Kubono, Kimiko Kado, Yukiyasu Kashiwagi, Keita Tani and Kunihiko Yokoi}

Computing details

Data collection: RAPID-AUTO (Rigaku, 2006); cell refinement: RAPID-AUTO (Rigaku, 2006); data reduction: RAPIDAUTO (Rigaku, 2006); program(s) used to solve structure: SIR92 (Altomare et al., 1993); program(s) used to refine structure: SHELXL97 (Sheldrick, 2008); molecular graphics: PLATON (Spek, 2009); software used to prepare material for publication: CrystalStructure (Rigaku, 2014).

7-\{[Bis(pyridin-2-ylmethyl)amino]methyl\}-5-chloroquinolin-8-ol

\section{Crystal data}

$\mathrm{C}_{22} \mathrm{H}_{19} \mathrm{ClN}_{4} \mathrm{O}$

$M_{r}=390.86$

Triclinic, $P \overline{1}$

$a=8.3170(5) \AA$

$b=11.5993(7) \AA$

$c=11.6135(6) \AA$

$\alpha=116.8473(13)^{\circ}$

$\beta=105.2809(13)^{\circ}$

$\gamma=92.0110(17)^{\circ}$

$V=948.68(10) \AA^{3}$

\section{Data collection}

Rigaku R-AXIS RAPID diffractometer

Detector resolution: 10.000 pixels $\mathrm{mm}^{-1}$ $\omega$ scans

Absorption correction: multi-scan

(ABSCOR; Higashi, 1995)

$T_{\min }=0.769, T_{\max }=0.978$

9412 measured reflections

\section{Refinement}

Refinement on $F^{2}$

$R\left[F^{2}>2 \sigma\left(F^{2}\right)\right]=0.036$

$w R\left(F^{2}\right)=0.123$

$S=1.09$

4293 reflections

257 parameters

0 restraints

Primary atom site location: structure-invariant direct methods

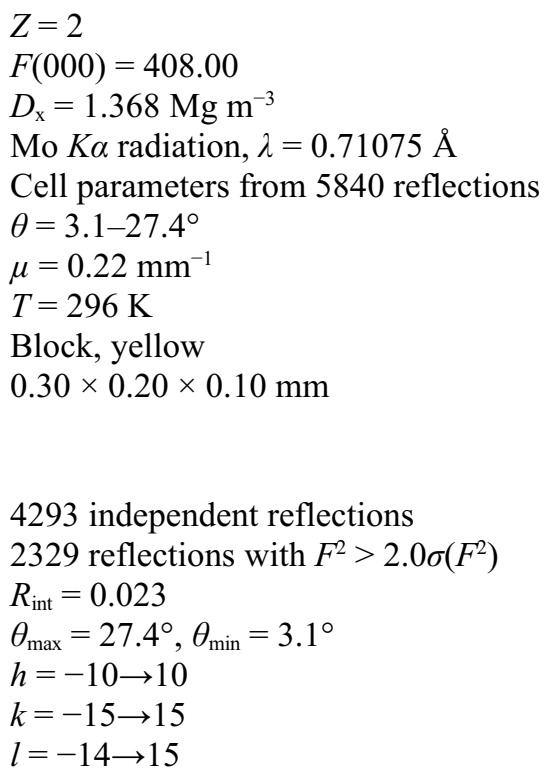

4293 independent reflections 2329 reflections with $F^{2}>2.0 \sigma\left(F^{2}\right)$

$R_{\text {int }}=0.023$

$\theta_{\max }=27.4^{\circ}, \theta_{\min }=3.1^{\circ}$

$h=-10 \rightarrow 10$

$k=-15 \rightarrow 15$

$l=-14 \rightarrow 15$

Secondary atom site location: difference Fourier map

Hydrogen site location: inferred from neighbouring sites

$\mathrm{H}$ atoms treated by a mixture of independent and constrained refinement

$w=1 /\left[\sigma^{2}\left(F_{\mathrm{o}}^{2}\right)+(0.0488 P)^{2}+0.1777 P\right]$ where $P=\left(F_{\mathrm{o}}{ }^{2}+2 F_{\mathrm{c}}{ }^{2}\right) / 3$

$(\Delta / \sigma)_{\max }<0.001$ 
$\Delta \rho_{\max }=0.26 \mathrm{e} \AA^{-3}$

$\Delta \rho_{\min }=-0.24$ e $\AA^{-3}$

Special details

Geometry. Refinement was performed using all reflections. The weighted $R$-factor $(w R)$ and goodness of fit (S) are based on $\mathrm{F}^{2} . R$-factor (gt) are based on $\mathrm{F}$. The threshold expression of $\mathrm{F}^{2}>2.0 \operatorname{sigma}\left(\mathrm{F}^{2}\right)$ is used only for calculating $R$-factor (gt).

Refinement. Refinement was performed using all reflections. The weighted $R$-factor $(w R)$ and goodness of fit $(S)$ are based on $F^{2}$. $R$-factor (gt) are based on $F$. The threshold expression of $F^{2}>2.0 \sigma\left(F^{2}\right)$ is used only for calculating $R$-factor (gt).

Fractional atomic coordinates and isotropic or equivalent isotropic displacement parameters $\left(\hat{A}^{2}\right)$

\begin{tabular}{|c|c|c|c|c|}
\hline & $x$ & $y$ & $z$ & $U_{\text {iso }} * / U_{\text {eq }}$ \\
\hline $\mathrm{C} 11$ & $0.20020(9)$ & $0.40251(6)$ & $0.58501(6)$ & $0.0724(2)$ \\
\hline $\mathrm{O} 2$ & $0.3787(2)$ & $0.45209(15)$ & $0.14798(14)$ & $0.0563(4)$ \\
\hline N3 & $0.3767(2)$ & $0.22221(18)$ & $0.14868(17)$ & $0.0560(5)$ \\
\hline N4 & $0.1525(2)$ & $0.72637(15)$ & $0.33905(15)$ & $0.0432(4)$ \\
\hline N5 & $0.2432(2)$ & $0.61932(17)$ & $0.06500(16)$ & $0.0500(4)$ \\
\hline N6 & $0.2990(2)$ & $1.07071(18)$ & $0.58796(17)$ & $0.0562(5)$ \\
\hline $\mathrm{C} 7$ & $0.3408(2)$ & $0.4468(2)$ & $0.25274(18)$ & $0.0444(5)$ \\
\hline $\mathrm{C} 8$ & $0.3075(2)$ & $0.5531(2)$ & 0.35655 (19) & $0.0440(5)$ \\
\hline C9 & $0.2637(3)$ & $0.5344(2)$ & $0.45812(19)$ & $0.0488(5)$ \\
\hline $\mathrm{C} 10$ & $0.2564(3)$ & $0.4177(2)$ & $0.45746(19)$ & $0.0474(5)$ \\
\hline C11 & $0.2949(2)$ & $0.3074(2)$ & $0.35528(18)$ & $0.0451(5)$ \\
\hline $\mathrm{C} 12$ & $0.2940(3)$ & $0.1827(2)$ & $0.3486(2)$ & $0.0546(5)$ \\
\hline $\mathrm{C} 13$ & $0.3335(3)$ & $0.0841(2)$ & $0.2454(2)$ & $0.0642(6)$ \\
\hline $\mathrm{C} 14$ & $0.3733(3)$ & $0.1088(2)$ & $0.1483(2)$ & $0.0645(6)$ \\
\hline $\mathrm{C} 15$ & $0.3387(2)$ & $0.3236(2)$ & $0.25179(18)$ & $0.0439(5)$ \\
\hline $\mathrm{C} 16$ & $0.3190(3)$ & $0.6889(2)$ & $0.3710(2)$ & $0.0469(5)$ \\
\hline $\mathrm{C} 17$ & $0.0484(3)$ & $0.6472(2)$ & $0.19651(18)$ & $0.0453(5)$ \\
\hline C18 & $0.1068(3)$ & $0.67040(19)$ & $0.09391(18)$ & $0.0440(5)$ \\
\hline C19 & $0.0266(3)$ & $0.7400(2)$ & $0.0336(2)$ & $0.0542(5)$ \\
\hline $\mathrm{C} 20$ & $0.0860(3)$ & $0.7593(3)$ & $-0.0582(2)$ & $0.0646(6)$ \\
\hline $\mathrm{C} 21$ & $0.2264(3)$ & $0.7087(3)$ & $-0.0865(2)$ & $0.0637(6)$ \\
\hline $\mathrm{C} 22$ & $0.2999(3)$ & $0.6399(2)$ & $-0.0238(2)$ & $0.0577(6)$ \\
\hline $\mathrm{C} 23$ & $0.1681(3)$ & $0.8665(2)$ & $0.3842(2)$ & $0.0549(6)$ \\
\hline $\mathrm{C} 24$ & $0.2147(3)$ & $0.9495(2)$ & 0.53687 (19) & $0.0463(5)$ \\
\hline $\mathrm{C} 25$ & $0.1679(3)$ & $0.9048(2)$ & $0.6174(2)$ & $0.0563(6)$ \\
\hline $\mathrm{C} 26$ & $0.2149(3)$ & $0.9865(2)$ & $0.7564(2)$ & $0.0600(6)$ \\
\hline $\mathrm{C} 27$ & $0.3044(3)$ & $1.1103(2)$ & $0.8101(2)$ & $0.0570(6)$ \\
\hline $\mathrm{C} 28$ & $0.3404(3)$ & $1.1481(2)$ & $0.7224(2)$ & $0.0614(6)$ \\
\hline $\mathrm{H} 2$ & $0.329(3)$ & $0.526(3)$ & $0.129(3)$ & $0.098(9)^{*}$ \\
\hline H9 & 0.23914 & 0.60515 & 0.52745 & $0.0585^{*}$ \\
\hline H12 & 0.26668 & 0.16817 & 0.41415 & $0.0655^{*}$ \\
\hline H13 & 0.33386 & 0.00152 & 0.23959 & $0.0771^{*}$ \\
\hline H14 & 0.39931 & 0.0399 & 0.07819 & $0.0774 *$ \\
\hline H16A & 0.38532 & 0.7515 & 0.46354 & $0.0563^{*}$ \\
\hline H16B & 0.37804 & 0.69349 & 0.31109 & $0.0563 *$ \\
\hline H17A & -0.06633 & 0.66426 & 0.18846 & $0.0544 *$ \\
\hline
\end{tabular}




\begin{tabular}{lllll} 
H17B & 0.04458 & 0.55522 & 0.17233 & $0.0544^{*}$ \\
H19 & -0.06765 & 0.77418 & 0.05442 & $0.0651^{*}$ \\
H20 & 0.03192 & 0.80564 & -0.10001 & $0.0776^{*}$ \\
H21 & 0.27022 & 0.72111 & -0.14678 & $0.0764^{*}$ \\
H22 & 0.39441 & 0.60539 & -0.04348 & $0.0692^{*}$ \\
H23A & 0.06141 & 0.88388 & 0.34197 & $0.0659^{*}$ \\
H23B & 0.25394 & 0.89247 & 0.35362 & $0.0659^{*}$ \\
H25 & 0.10548 & 0.82092 & 0.57873 & $0.0676^{*}$ \\
H26 & 0.18619 & 0.95777 & 0.81246 & $0.0720^{*}$ \\
H27 & 0.33951 & 1.16686 & 0.90321 & $0.0684^{*}$ \\
H28 & 0.39751 & 1.2333 & 0.75867 & $0.0737^{*}$ \\
\hline
\end{tabular}

Atomic displacement parameters $\left(\AA^{2}\right)$

\begin{tabular}{|c|c|c|c|c|c|c|}
\hline & $U^{11}$ & $U^{22}$ & $U^{33}$ & $U^{12}$ & $U^{13}$ & $U^{23}$ \\
\hline $\mathrm{Cl1}$ & $0.0990(5)$ & $0.0762(4)$ & $0.0553(4)$ & 0.0150 & $0.0451(3)$ & $0.0312(3)$ \\
\hline $\mathrm{O} 2$ & $0.0698(10)$ & $0.0665(10)$ & $0.0499(8)$ & $0.0272(8)$ & $0.0355(8)$ & $0.0321(8)$ \\
\hline N3 & $0.0734(13)$ & $0.0579(12)$ & $0.0446(10)$ & $0.0263(10)$ & $0.0282(9)$ & $0.0247(9)$ \\
\hline $\mathrm{N} 4$ & $0.0515(10)$ & $0.0396(9)$ & $0.0336(8)$ & $0.0077(8)$ & $0.0159(7)$ & $0.0123(8)$ \\
\hline N5 & $0.0525(11)$ & $0.0546(11)$ & $0.0389(9)$ & $0.0099(9)$ & $0.0180(8)$ & $0.0169(9)$ \\
\hline N6 & $0.0747(13)$ & $0.0454(11)$ & $0.0413(10)$ & $0.0005(9)$ & $0.0243(9)$ & $0.0122(9)$ \\
\hline C7 & $0.0422(11)$ & $0.0567(13)$ & $0.0350(10)$ & $0.0116(10)$ & $0.0153(9)$ & $0.0206(10)$ \\
\hline $\mathrm{C} 8$ & $0.0415(11)$ & $0.0493(12)$ & $0.0364(10)$ & $0.0063(9)$ & $0.0121(8)$ & $0.0167(9)$ \\
\hline C9 & $0.0504(12)$ & $0.0543(13)$ & $0.0336(10)$ & $0.0101(10)$ & $0.0165(9)$ & $0.0125(10)$ \\
\hline $\mathrm{C} 10$ & $0.0511(12)$ & $0.0551(14)$ & $0.0363(10)$ & $0.0075(10)$ & $0.0161(9)$ & $0.0208(10)$ \\
\hline $\mathrm{C} 11$ & $0.0416(11)$ & $0.0543(13)$ & $0.0347(10)$ & $0.0079(10)$ & $0.0108(8)$ & $0.0181(10)$ \\
\hline $\mathrm{C} 12$ & $0.0622(14)$ & $0.0598(15)$ & $0.0463(12)$ & $0.0117(11)$ & $0.0188(11)$ & $0.0281(12)$ \\
\hline $\mathrm{C} 13$ & $0.0846(18)$ & $0.0573(15)$ & $0.0593(14)$ & $0.0216(13)$ & $0.0287(13)$ & $0.0308(13)$ \\
\hline $\mathrm{C} 14$ & $0.0896(18)$ & $0.0579(15)$ & $0.0530(13)$ & $0.0316(13)$ & $0.0333(13)$ & $0.0250(12)$ \\
\hline C15 & $0.0437(11)$ & $0.0522(13)$ & $0.0352(10)$ & $0.0132(10)$ & $0.0143(9)$ & $0.0192(10)$ \\
\hline C16 & $0.0478(12)$ & $0.0478(12)$ & $0.0364(10)$ & $0.0022(10)$ & $0.0140(9)$ & $0.0129(9)$ \\
\hline $\mathrm{C} 17$ & $0.0450(11)$ & $0.0461(12)$ & $0.0361(10)$ & $0.0051(9)$ & $0.0135(9)$ & $0.0122(9)$ \\
\hline $\mathrm{C} 18$ & $0.0441(11)$ & $0.0431(11)$ & $0.0305(9)$ & $0.0027(9)$ & $0.0095(8)$ & $0.0075(9)$ \\
\hline C19 & $0.0544(13)$ & $0.0585(14)$ & $0.0441(11)$ & $0.0130(11)$ & $0.0146(10)$ & $0.0201(11)$ \\
\hline $\mathrm{C} 20$ & $0.0732(17)$ & $0.0701(16)$ & $0.0520(13)$ & $0.0108(13)$ & $0.0155(12)$ & $0.0326(13)$ \\
\hline $\mathrm{C} 21$ & $0.0717(16)$ & $0.0737(17)$ & $0.0466(12)$ & $0.0020(13)$ & $0.0214(12)$ & $0.0288(13)$ \\
\hline $\mathrm{C} 22$ & $0.0566(14)$ & $0.0681(15)$ & $0.0446(12)$ & $0.0090(12)$ & $0.0230(10)$ & $0.0203(12)$ \\
\hline $\mathrm{C} 23$ & $0.0801(16)$ & $0.0432(13)$ & $0.0376(11)$ & $0.0105(11)$ & $0.0217(11)$ & $0.0144(10)$ \\
\hline $\mathrm{C} 24$ & $0.0585(13)$ & $0.0413(12)$ & $0.0363(10)$ & $0.0111(10)$ & $0.0191(9)$ & $0.0137(9)$ \\
\hline $\mathrm{C} 25$ & $0.0781(16)$ & $0.0463(13)$ & $0.0465(12)$ & $0.0078(11)$ & $0.0280(11)$ & $0.0193(11)$ \\
\hline $\mathrm{C} 26$ & $0.0820(17)$ & $0.0648(16)$ & $0.0449(12)$ & $0.0187(13)$ & $0.0331(12)$ & $0.0283(12)$ \\
\hline $\mathrm{C} 27$ & $0.0613(14)$ & $0.0620(15)$ & 0.0355 (11) & $0.0108(12)$ & $0.0189(10)$ & $0.0113(11)$ \\
\hline $\mathrm{C} 28$ & $0.0685(16)$ & $0.0535(14)$ & $0.0442(12)$ & $-0.0039(12)$ & $0.0215(11)$ & $0.0076(11)$ \\
\hline
\end{tabular}

Geometric parameters $\left(\AA,^{\circ}\right)$

\begin{tabular}{llll}
\hline $\mathrm{C} 11-\mathrm{C} 10$ & $1.743(3)$ & $\mathrm{C} 21-\mathrm{C} 22$ & $1.366(4)$ \\
$\mathrm{O} 2-\mathrm{C} 7$ & $1.361(3)$ & $\mathrm{C} 23-\mathrm{C} 24$ & $1.514(3)$
\end{tabular}




\begin{tabular}{|c|c|c|c|}
\hline $\mathrm{N} 3-\mathrm{C} 14$ & $1.313(4)$ & $\mathrm{C} 24-\mathrm{C} 25$ & $1.382(4)$ \\
\hline $\mathrm{N} 3-\mathrm{C} 15$ & $1.368(3)$ & $\mathrm{C} 25-\mathrm{C} 26$ & $1.384(3)$ \\
\hline $\mathrm{N} 4-\mathrm{C} 16$ & $1.470(3)$ & $\mathrm{C} 26-\mathrm{C} 27$ & $1.369(4)$ \\
\hline $\mathrm{N} 4-\mathrm{C} 17$ & $1.466(2)$ & $\mathrm{C} 27-\mathrm{C} 28$ & $1.370(4)$ \\
\hline $\mathrm{N} 4-\mathrm{C} 23$ & $1.454(3)$ & $\mathrm{O} 2-\mathrm{H} 2$ & $1.04(3)$ \\
\hline $\mathrm{N} 5-\mathrm{C} 18$ & $1.349(3)$ & C9-H9 & 0.930 \\
\hline $\mathrm{N} 5-\mathrm{C} 22$ & $1.347(4)$ & $\mathrm{C} 12-\mathrm{H} 12$ & 0.930 \\
\hline $\mathrm{N} 6-\mathrm{C} 24$ & $1.334(3)$ & $\mathrm{C} 13-\mathrm{H} 13$ & 0.930 \\
\hline $\mathrm{N} 6-\mathrm{C} 28$ & $1.338(3)$ & C14-H14 & 0.930 \\
\hline $\mathrm{C} 7-\mathrm{C} 8$ & $1.381(3)$ & $\mathrm{C} 16-\mathrm{H} 16 \mathrm{~A}$ & 0.970 \\
\hline $\mathrm{C} 7-\mathrm{C} 15$ & $1.424(4)$ & $\mathrm{C} 16-\mathrm{H} 16 \mathrm{~B}$ & 0.970 \\
\hline $\mathrm{C} 8-\mathrm{C} 9$ & $1.422(4)$ & C17-H17A & 0.970 \\
\hline $\mathrm{C} 8-\mathrm{C} 16$ & $1.504(3)$ & C17-H17B & 0.970 \\
\hline $\mathrm{C} 9-\mathrm{C} 10$ & $1.349(4)$ & C19-H19 & 0.930 \\
\hline $\mathrm{C} 10-\mathrm{C} 11$ & $1.416(3)$ & $\mathrm{C} 20-\mathrm{H} 20$ & 0.930 \\
\hline $\mathrm{C} 11-\mathrm{C} 12$ & $1.412(4)$ & $\mathrm{C} 21-\mathrm{H} 21$ & 0.930 \\
\hline $\mathrm{C} 11-\mathrm{C} 15$ & $1.429(4)$ & $\mathrm{C} 22-\mathrm{H} 22$ & 0.930 \\
\hline $\mathrm{C} 12-\mathrm{C} 13$ & $1.359(3)$ & $\mathrm{C} 23-\mathrm{H} 23 \mathrm{~A}$ & 0.970 \\
\hline $\mathrm{C} 13-\mathrm{C} 14$ & $1.394(5)$ & $\mathrm{C} 23-\mathrm{H} 23 \mathrm{~B}$ & 0.970 \\
\hline $\mathrm{C} 17-\mathrm{C} 18$ & $1.521(4)$ & $\mathrm{C} 25-\mathrm{H} 25$ & 0.930 \\
\hline $\mathrm{C} 18-\mathrm{C} 19$ & $1.375(4)$ & $\mathrm{C} 26-\mathrm{H} 26$ & 0.930 \\
\hline $\mathrm{C} 19-\mathrm{C} 20$ & $1.384(4)$ & $\mathrm{C} 27-\mathrm{H} 27$ & 0.930 \\
\hline $\mathrm{C} 20-\mathrm{C} 21$ & $1.376(4)$ & $\mathrm{C} 28-\mathrm{H} 28$ & 0.930 \\
\hline $\mathrm{C} 14-\mathrm{N} 3-\mathrm{C} 15$ & $117.7(2)$ & $\mathrm{N} 6-\mathrm{C} 28-\mathrm{C} 27$ & $124.4(2)$ \\
\hline $\mathrm{C} 16-\mathrm{N} 4-\mathrm{C} 17$ & $113.69(15)$ & $\mathrm{C} 7-\mathrm{O} 2-\mathrm{H} 2$ & $112.4(18)$ \\
\hline $\mathrm{C} 16-\mathrm{N} 4-\mathrm{C} 23$ & $111.51(16)$ & $\mathrm{C} 8-\mathrm{C} 9-\mathrm{H} 9$ & 118.825 \\
\hline $\mathrm{C} 17-\mathrm{N} 4-\mathrm{C} 23$ & $112.33(18)$ & $\mathrm{C} 10-\mathrm{C} 9-\mathrm{H} 9$ & 118.839 \\
\hline $\mathrm{C} 18-\mathrm{N} 5-\mathrm{C} 22$ & $117.9(2)$ & $\mathrm{C} 11-\mathrm{C} 12-\mathrm{H} 12$ & 120.293 \\
\hline $\mathrm{C} 24-\mathrm{N} 6-\mathrm{C} 28$ & $117.2(2)$ & $\mathrm{C} 13-\mathrm{C} 12-\mathrm{H} 12$ & 120.294 \\
\hline $\mathrm{O} 2-\mathrm{C} 7-\mathrm{C} 8$ & $123.5(2)$ & $\mathrm{C} 12-\mathrm{C} 13-\mathrm{H} 13$ & 120.515 \\
\hline $\mathrm{O} 2-\mathrm{C} 7-\mathrm{C} 15$ & $116.17(17)$ & $\mathrm{C} 14-\mathrm{C} 13-\mathrm{H} 13$ & 120.514 \\
\hline $\mathrm{C} 8-\mathrm{C} 7-\mathrm{C} 15$ & $120.4(2)$ & N3-C14-H14 & 117.649 \\
\hline $\mathrm{C} 7-\mathrm{C} 8-\mathrm{C} 9$ & $118.4(2)$ & $\mathrm{C} 13-\mathrm{C} 14-\mathrm{H} 14$ & 117.655 \\
\hline $\mathrm{C} 7-\mathrm{C} 8-\mathrm{C} 16$ & $124.0(2)$ & $\mathrm{N} 4-\mathrm{C} 16-\mathrm{H} 16 \mathrm{~A}$ & 108.956 \\
\hline $\mathrm{C} 9-\mathrm{C} 8-\mathrm{C} 16$ & $117.66(18)$ & $\mathrm{N} 4-\mathrm{C} 16-\mathrm{H} 16 \mathrm{~B}$ & 108.959 \\
\hline $\mathrm{C} 8-\mathrm{C} 9-\mathrm{C} 10$ & $122.34(19)$ & $\mathrm{C} 8-\mathrm{C} 16-\mathrm{H} 16 \mathrm{~A}$ & 108.959 \\
\hline $\mathrm{C} 11-\mathrm{C} 10-\mathrm{C} 9$ & $119.55(16)$ & $\mathrm{C} 8-\mathrm{C} 16-\mathrm{H} 16 \mathrm{~B}$ & 108.962 \\
\hline $\mathrm{C} 11-\mathrm{C} 10-\mathrm{C} 11$ & $119.4(2)$ & $\mathrm{H} 16 \mathrm{~A}-\mathrm{C} 16-\mathrm{H} 16 \mathrm{~B}$ & 107.759 \\
\hline $\mathrm{C} 9-\mathrm{C} 10-\mathrm{C} 11$ & $121.1(2)$ & $\mathrm{N} 4-\mathrm{C} 17-\mathrm{H} 17 \mathrm{~A}$ & 108.296 \\
\hline $\mathrm{C} 10-\mathrm{C} 11-\mathrm{C} 12$ & $124.8(2)$ & N4-C17-H17B & 108.295 \\
\hline $\mathrm{C} 10-\mathrm{C} 11-\mathrm{C} 15$ & $117.6(2)$ & $\mathrm{C} 18-\mathrm{C} 17-\mathrm{H} 17 \mathrm{~A}$ & 108.301 \\
\hline $\mathrm{C} 12-\mathrm{C} 11-\mathrm{C} 15$ & $117.59(18)$ & $\mathrm{C} 18-\mathrm{C} 17-\mathrm{H} 17 \mathrm{~B}$ & 108.301 \\
\hline $\mathrm{C} 11-\mathrm{C} 12-\mathrm{C} 13$ & $119.4(3)$ & $\mathrm{H} 17 \mathrm{~A}-\mathrm{C} 17-\mathrm{H} 17 \mathrm{~B}$ & 107.402 \\
\hline $\mathrm{C} 12-\mathrm{C} 13-\mathrm{C} 14$ & $119.0(3)$ & $\mathrm{C} 18-\mathrm{C} 19-\mathrm{H} 19$ & 119.991 \\
\hline $\mathrm{N} 3-\mathrm{C} 14-\mathrm{C} 13$ & $124.7(2)$ & $\mathrm{C} 20-\mathrm{C} 19-\mathrm{H} 19$ & 119.983 \\
\hline $\mathrm{N} 3-\mathrm{C} 15-\mathrm{C} 7$ & $118.2(2)$ & $\mathrm{C} 19-\mathrm{C} 20-\mathrm{H} 20$ & 120.561 \\
\hline $\mathrm{N} 3-\mathrm{C} 15-\mathrm{C} 11$ & $121.6(2)$ & $\mathrm{C} 21-\mathrm{C} 20-\mathrm{H} 20$ & 120.563 \\
\hline
\end{tabular}




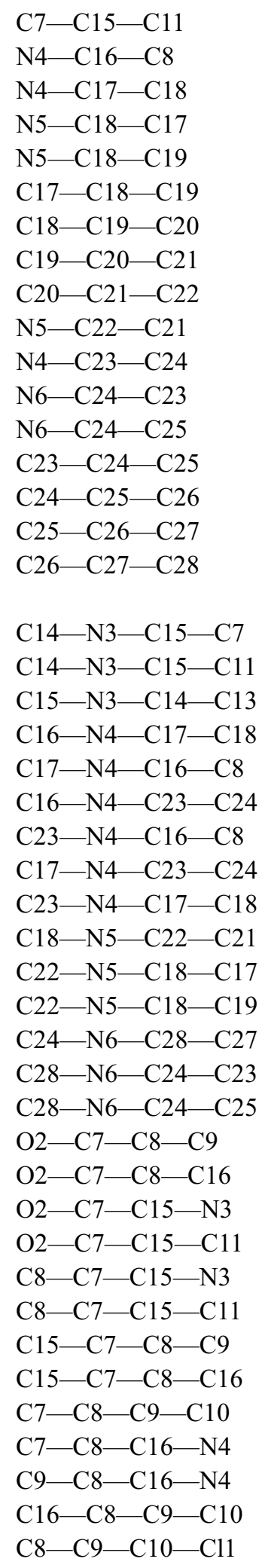

$120.18(18)$

$113.11(17)$

115.94 (17)

116.4 (2)

$121.2(2)$

122.4 (2)

$120.0(2)$

118.9 (3)

$118.3(3)$

$123.7(2)$

$113.4(2)$

$115.3(2)$

$122.17(18)$

$122.53(19)$

$119.2(2)$

119.0 (3)

117.95 (19)

$-179.57(17)$

$-0.6(3)$

0.1 (3)

$-70.7(2)$

$-65.3(2)$

$-72.8(2)$

$166.49(16)$

$158.23(17)$

57.1 (2)

-0.4 (2)

$-178.93(13)$

$0.8(2)$

$1.1(3)$

$178.99(18)$

$1.3(3)$

$177.87(14)$

$-3.8(3)$

$1.0(2)$

$-177.99(13)$

$-178.35(15)$

$2.7(2)$

$-2.8(2)$

$175.54(14)$

$1.1(3)$

$107.8(2)$

$-73.8(2)$

$-177.37(15)$

$-179.55(14)$

$\begin{array}{ll}\mathrm{C} 20-\mathrm{C} 21-\mathrm{H} 21 & 120.849 \\ \mathrm{C} 22-\mathrm{C} 21-\mathrm{H} 21 & 120.854 \\ \mathrm{~N} 5-\mathrm{C} 22-\mathrm{H} 22 & 118.151 \\ \mathrm{C} 21-\mathrm{C} 22-\mathrm{H} 22 & 118.151 \\ \mathrm{~N} 4-\mathrm{C} 23-\mathrm{H} 23 \mathrm{~A} & 108.897 \\ \mathrm{~N} 4-\mathrm{C} 23-\mathrm{H} 23 \mathrm{~B} & 108.899 \\ \mathrm{C} 24-\mathrm{C} 23-\mathrm{H} 23 \mathrm{~A} & 108.892 \\ \mathrm{C} 24-\mathrm{C} 23-\mathrm{H} 23 \mathrm{~B} & 108.896 \\ \mathrm{H} 23 \mathrm{~A}-\mathrm{C} 23-\mathrm{H} 23 \mathrm{~B} & 107.725 \\ \mathrm{C} 24-\mathrm{C} 25-\mathrm{H} 25 & 120.400 \\ \mathrm{C} 26-\mathrm{C} 25-\mathrm{H} 25 & 120.399 \\ \mathrm{C} 25-\mathrm{C} 26-\mathrm{H} 26 & 120.492 \\ \mathrm{C} 27-\mathrm{C} 26-\mathrm{H} 26 & 120.490 \\ \mathrm{C} 26-\mathrm{C} 27-\mathrm{H} 27 & 121.022 \\ \mathrm{C} 28-\mathrm{C} 27-\mathrm{H} 27 & 121.032 \\ \mathrm{~N} 6-\mathrm{C} 28-\mathrm{H} 28 & 117.816 \\ \mathrm{C} 27-\mathrm{C} 28-\mathrm{H} 28 & 117.809\end{array}$

$\mathrm{C} 8-\mathrm{C} 9-\mathrm{C} 10-\mathrm{C} 11$

$\mathrm{C} 11-\mathrm{C} 10-\mathrm{C} 11-\mathrm{C} 12 \quad-1.0(2)$

$\mathrm{C} 11-\mathrm{C} 10-\mathrm{C} 11-\mathrm{C} 15 \quad 179.37$ (11)

$\mathrm{C} 9-\mathrm{C} 10-\mathrm{C} 11-\mathrm{C} 12 \quad 178.57$ (16)

$\mathrm{C} 9-\mathrm{C} 10-\mathrm{C} 11-\mathrm{C} 15 \quad-1.1$ (3)

$\mathrm{C} 10-\mathrm{C} 11-\mathrm{C} 12-\mathrm{C} 13 \quad-179.91(16)$

$\mathrm{C} 10-\mathrm{C} 11-\mathrm{C} 15-\mathrm{N} 3 \quad-179.63(15)$

$\mathrm{C} 10-\mathrm{C} 11-\mathrm{C} 15-\mathrm{C} 7 \quad-0.7$ (2)

$\mathrm{C} 12-\mathrm{C} 11-\mathrm{C} 15-\mathrm{N} 3 \quad 0.7$ (2)

$\mathrm{C} 12-\mathrm{C} 11-\mathrm{C} 15-\mathrm{C} 7 \quad 179.66(15)$

$\mathrm{C} 15-\mathrm{C} 11-\mathrm{C} 12-\mathrm{C} 13 \quad-0.3(3)$

$\mathrm{C} 11-\mathrm{C} 12-\mathrm{C} 13-\mathrm{C} 14 \quad-0.2(3)$

$\mathrm{C} 12-\mathrm{C} 13-\mathrm{C} 14-\mathrm{N} 3 \quad 0.4(4)$

$\mathrm{N} 4-\mathrm{C} 17-\mathrm{C} 18-\mathrm{N} 5 \quad 75.0$ (2)

$\mathrm{N} 4-\mathrm{C} 17-\mathrm{C} 18-\mathrm{C} 19 \quad-104.73$ (19)

$\mathrm{N} 5-\mathrm{C} 18-\mathrm{C} 19-\mathrm{C} 20 \quad-0.3$ (2)

$\mathrm{C} 17-\mathrm{C} 18-\mathrm{C} 19-\mathrm{C} 20 \quad 179.38$ (13)

$\mathrm{C} 18-\mathrm{C} 19-\mathrm{C} 20-\mathrm{C} 21-0.5$ (3)

$\mathrm{C} 19-\mathrm{C} 20-\mathrm{C} 21-\mathrm{C} 22 \quad 0.9(3)$

$\mathrm{C} 20-\mathrm{C} 21-\mathrm{C} 22-\mathrm{N} 5 \quad-0.4$ (3)

$\mathrm{N} 4-\mathrm{C} 23-\mathrm{C} 24-\mathrm{N} 6$

$\mathrm{N} 4-\mathrm{C} 23-\mathrm{C} 24-\mathrm{C} 25$

$152.46(19)$

$-29.9(3)$

$-2.4(4)$

$-179.9(2)$

$1.0(4)$

$1.2(4)$

$-2.3(4)$ 
Hydrogen-bond geometry $\left(A,{ }^{\circ}\right)$

$\mathrm{Cg} 2$ and $\mathrm{Cg} 3$ are the centroids of rings $\mathrm{N} 5 / \mathrm{C} 18-\mathrm{C} 22$ and $\mathrm{N} 6 / \mathrm{C} 24-\mathrm{C} 28$, respectively.

\begin{tabular}{lllll}
\hline$D-\mathrm{H} \cdots A$ & $D-\mathrm{H}$ & $\mathrm{H} \cdots A$ & $D \cdots A$ & $D-\mathrm{H} \cdots A$ \\
\hline $\mathrm{O} 2-\mathrm{H} 2 \cdots \mathrm{N} 5$ & $1.04(3)$ & $1.66(4)$ & $2.689(3)$ & $168(2)$ \\
$\mathrm{C} 22-\mathrm{H} 22 \cdots \mathrm{O} 2^{\mathrm{i}}$ & 0.93 & 2.46 & $3.348(3)$ & 160 \\
$\mathrm{C} 27-\mathrm{H} 27 \cdots \mathrm{N}^{\mathrm{ii}}$ & 0.93 & 2.55 & $3.406(3)$ & 153 \\
$\mathrm{C} 17-\mathrm{H} 17 \mathrm{~b} \cdots \mathrm{Cg}^{2 i i}$ & 0.97 & 2.79 & $3.599(3)$ & 141 \\
$\mathrm{C} 23-\mathrm{H} 23 A \cdots \mathrm{Cg}^{\mathrm{ii}}$ & 0.97 & 2.86 & $3.770(3)$ & 156
\end{tabular}

Symmetry codes: (i) $-x+1,-y+1,-z$; (ii) $x, y+1, z+1$; (iii) $-x,-y+1,-z$; (iv) $-x,-y+2,-z+1$. 